\title{
REGIONAL OFFSET OF RADIOCARBON CONCENTRATION AND ITS VARIATION IN THE KOREAN ATMOSPHERE FROM AD 1650-1850
}

\author{
Wan Hong ${ }^{1,2}$ • Jung Hun Park ${ }^{1} \cdot$ Gyujun Park ${ }^{1} \cdot$ Ki Suk Sung $\bullet$ Won Kyu Park ${ }^{3}$ Jong-Geol Lee ${ }^{1}$ \\ ABSTRACT. A series of annual tree-ring measurements has been performed in order to reconstruct the radiocarbon concen- \\ tration variation in the Korean atmosphere from AD 1650 to 1850. The absolute ages of the samples were determined using \\ dendrochronology. Alpha-cellulose extraction was applied to prepare the tree-ring samples for precise ${ }^{14} \mathrm{C}$ measurement. The \\ ${ }^{14} \mathrm{C}$ concentrations of the tree rings were then plotted with the dendrochronological ages and showed that during the period \\ AD 1650-1850, the discrepancy in ${ }^{14} \mathrm{C}$ concentration in the Korean atmosphere from IntCal data is small enough to use IntCal \\ data without any further correction. This is nearly one third of the average offset of the $400 \mathrm{yr}$ from AD 1250 to 1650 . One \\ of the probable causes for the regional offset around Korea is the contribution of ${ }^{14} \mathrm{C}$-depleted $\mathrm{CO}_{2}$ released from the northern \\ Pacific Ocean, where old deep water upwells to the surface. It is likely that the release rate of ${ }^{14} \mathrm{C}$-depleted $\mathrm{CO}_{2}$ decreased due \\ to the temperature change during the Little Ice Age.
}

\section{INTRODUCTION}

Radiocarbon concentration variations in the atmosphere depend on variations of natural activities such as the ${ }^{14} \mathrm{C}$ production rate, climate change, and volcanic eruptions, as well as anthropogenic behaviors such as fossil fuel consumption and nuclear bomb tests. Among these factors, volcanic eruptions and local fossil fuel releases can cause regional offsets in ${ }^{14} \mathrm{C}$ concentrations. In particular, the contribution of $\mathrm{CO}_{2}$ released from the deep ocean is notable because oceans contain old carbon due to the long periods of seawater circulation. Previous studies reported that the ${ }^{14} \mathrm{C}$ concentrations in tree rings grown in east Asia are slightly lower than those of western Europe and North America (Nakamura et al. 2007; Hong et al. 2013). It appears that the low concentration is due to the migration of ${ }^{14} \mathrm{C}$-depleted $\mathrm{CO}_{2}$ into the Korean and Japanese atmosphere when it is released from the northern Pacific Ocean. The calendar age obtained by calibration using IntCal09 data (Reimer et al. 2009) may include offsets for the samples collected in the east Asian region because IntCal04 data after 12.4 cal kyr BP were obtained from tree-ring measurements of trees grown in Europe and North America, despite the fact that the IntCal04 data were provided after very careful crosschecking with European tree rings (German pines, German oaks, Belfast oaks, and Irish oaks) and North American rings (from Washington, Oregon, California and Alaska and bristlecone pine trees) (Reimer et al. 2004). To confirm the consistency in IntCal data used for calibrating the ${ }^{14} \mathrm{C}$ ages of local samples in the Far East, careful and precise ${ }^{14} \mathrm{C}$ concentration measurements of tree rings grown in this area are very important.

Since 2009, the 1MV accelerator mass spectrometry (AMS) system of the Korea Institute of Geoscience and Mineral Resources (KIGAM) has been dedicated to ${ }^{14} \mathrm{C}$ measurements of tree rings grown on the Korean Peninsula in order to study the regional characteristics of atmospheric isotopic concentration. ${ }^{14} \mathrm{C}$ variations of tree-ring samples that grew in Korea from AD 1250 to 1650 were initially reported at the 12th AMS Conference held in Wellington (Hong et al. 2013). This article builds on that work and reports ${ }^{14} \mathrm{C}$ concentrations of tree rings spanning the next $200 \mathrm{yr}$, from AD 1650 to 1850.

\footnotetext{
${ }^{1}$ Korea Institute of Geoscience and Mineral Resources (KIGAM), 124 Gwahang-no, Yuseong-gu, Daejeon 305-350, Republic of Korea.

${ }^{2}$ Corresponding author. Email: whong@kigam.re.kr.

${ }^{3}$ Tree-Ring Material Bank, Chungbuk National University, 52 Naesoodong-Ro, Heungdeok-gu, Cheongju, Chungbuk 361763, Republic of Korea.
} 


\section{W Hong et al.}

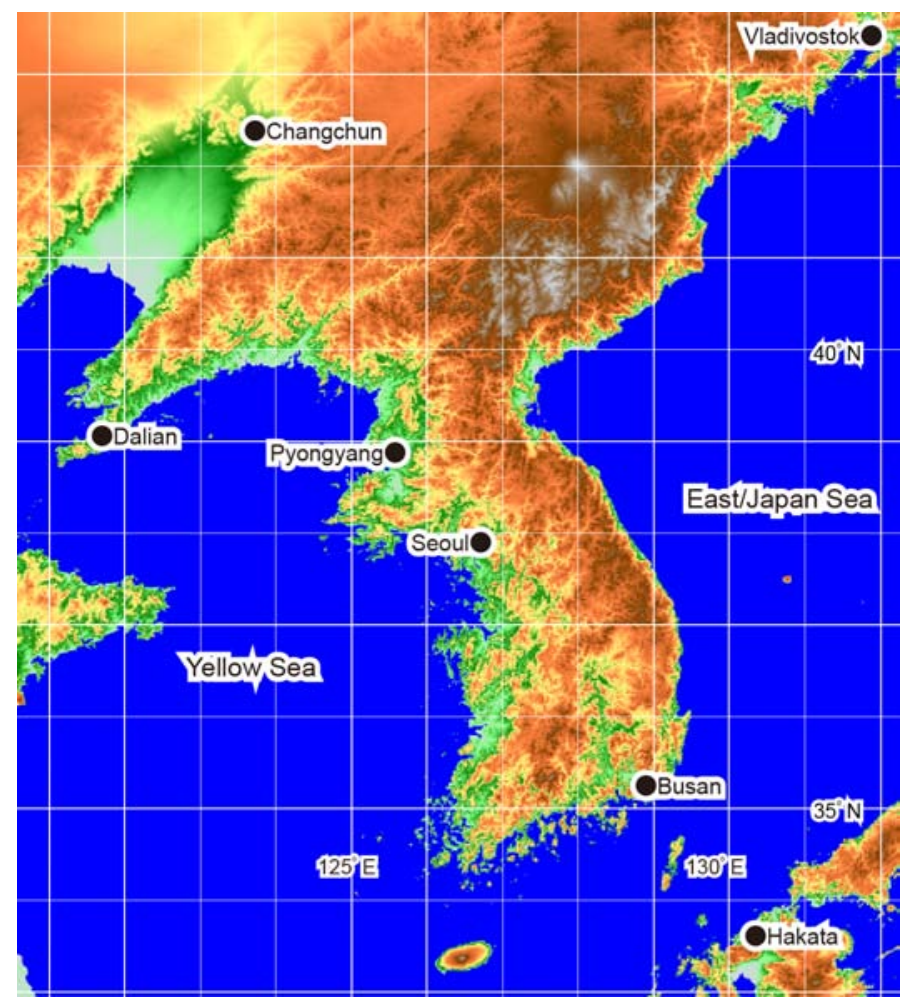

Figure 1 The location of the sampling sites in the Korean Peninsula

\section{SAMPLE COLLECTION AND DENDROCHRONOLOGY}

The tree-ring samples used in this work were collected from Tongmyeongjeon $\left(37^{\circ} 34^{\prime} 46.98^{\prime \prime} \mathrm{N}\right.$, $126^{\circ} 59^{\prime} 37.60^{\prime \prime} E$; see Figure 1), which is an annex of the Changdeok Palace in Seoul. This building was built in AD 1484 during the Choseon Dynasty, the last dynasty in Korea. In 2002, Tongmyeongjeon was renovated and wooden building materials were collected. Among them, 3 samples (TMJS017A, TMJS079A, and TMgt215A) were used for tree-ring measurements of pine wood (Pinus densiflora: Japanese red pine). Dendrochronological ages of the tree-ring samples were obtained from the Tree-Ring Material Bank of Chungbuk National University using Baillie's method (Baillie 1982). The ring-width plots of the samples were cross-dated by matching their patterns with the master chronologies that had already been absolutely dated through matches with living trees. The correlation coefficient $r$ between the sample and its reference is given as

$$
r=\frac{\sum\left[\left(S_{i}-S\right)\left(R_{i}-R\right)\right]}{\sqrt{\sum\left[\left(S_{i}-S\right)^{2}\left(R_{i}-R\right)^{2}\right]}}
$$

where $S_{i}$ is the $i^{\text {th }}$ ring width of a sample, $S$ is the average ring width of the sample, $R_{i}$ is the $i^{\text {th }}$ ring width of a reference, and $R$ is the average ring width of the reference (Baillie and Pilcher 1973). The $t$ value in the dating results is therefore defined as

$$
t=\frac{r \sqrt{n-2}}{1-r^{2}}
$$




\section{Regional Offset \& Variation in Korean Atmosphere}

where $n$ is the number of overlapped years in both the sample and reference. The age range of TMJS017A is 186 yr (AD 1619-1804). The master chronology for this sample was P3P1001M, and the $t$ value was 5.9. TMJS079A was a roof packing log with an age range of $135 \mathrm{yr}$ (AD 16991833). The master for this was KNNNUNP1, and the $t$ value was 6.7. Another roof packing log, TMgt215A, was found to have an age range of $139 \mathrm{yr}$ (AD 1757-1895) using the master, SINSUNP1. Its $t$ value was calculated to be 9.5. The sample information is summarized in Table 1 . Tree-ring chronologies of Japanese red pine in Korea, which were used as masters, were described in previous papers (Park and Lee 2001; Park et al. 2007).

Table 1 List of samples used for tree-ring measurements and chronology masters. $t$ values were calculated using the formula given by Baillie and Pilcher (1973).

\begin{tabular}{lllll}
\hline Sample & Master & Age range & Ranges used in this work & $t$ value \\
\hline TMJS017A & P3P1001M & AD 1619-1804 (186 yr) & AD 1650-1749 (100 yr) & 5.9 \\
TMJS079A & KNNNUNP1 & AD 1699-1833 (135 yr) & AD 1750-1790 (41 yr) & 6.7 \\
TMgt215A & SINSUNP1 & AD 1757-1895 (139 yr) & AD 1791-1850 (60 yr) & 9.5 \\
\hline
\end{tabular}

\section{SAMPLE PREPARATION AND MEASUREMENT OF RADIOCARBON}

After obtaining the absolute ages using dendrochronology, the samples were cut into annual rings to perform single-year measurements. An alpha-cellulose extraction procedure was utilized on 201 samples with ages from AD 1650 to 1850 . Each tree ring was powdered by a mixer mill with a diameter smaller than $0.5 \mathrm{~mm}$. The tree-ring powders were heated to $80^{\circ} \mathrm{C}$ in a Soxhlet system with a mixed solution of $120 \mathrm{~mL}$ of cyclohexane and $60 \mathrm{~mL}$ of ethanol for $6 \mathrm{hr}$ to remove gums, resins, waxes, sugars, oils, starches, alkaloids, tannins, and fats, followed by rinsing at $90{ }^{\circ} \mathrm{C}$ in a Soxhlet system with $180 \mathrm{~mL}$ of ethanol for $6 \mathrm{hr}$ to remove cyclohexane, which contains dead carbon, and a final rinse at $100^{\circ} \mathrm{C}$ in a Soxhlet containing $180 \mathrm{~mL}$ of deionized water for $6 \mathrm{hr}$ to remove ethanol, which may contain modern carbon. After the rinsing process, the samples were moved to flasks with a solution of $105 \mathrm{mg}$ of $\mathrm{NaClO}_{2}$ in $50 \mathrm{~mL}$ of $1 \mathrm{M} \mathrm{HCl}$ solvent, and the flasks were heated to $100{ }^{\circ} \mathrm{C}$ in an ultrasonic water bath for $1 \mathrm{hr}$ to remove lignin from the samples. During this step, the sample color typically changes to light brown. When the sample color was still dark, $50 \mathrm{mg}$ of $\mathrm{NaClO}_{2}$ was added to the solution, followed by additional heating for $1 \mathrm{hr}$. The samples were then rinsed with deionized water until neutral. Alpha-cellulose was extracted from the samples by an ultrasonic treatment at $60^{\circ} \mathrm{C}$ for $1 \mathrm{hr}$ in $50 \mathrm{~mL}$ of a $12 \% \mathrm{NaOH}$ solution with nitrogen bubbling. The solution was changed to a $7 \% \mathrm{NaOH}$ solution and the same procedure was repeated. The alpha-cellulose was rinsed so that it was neutralized. To remove any atmospheric $\mathrm{CO}_{2}$ contamination during the extraction process, the alpha-cellulose was treated with $2 \mathrm{M} \mathrm{HCl}$ at room temperature for $30 \mathrm{~min}$. Finally, the alpha-cellulose was rinsed with deionized water until neutral, after which it was dried at $40{ }^{\circ} \mathrm{C}$ for 2 days. All of these processes took a total of 5 days.

The conversion of alpha-cellulose to graphite was done using an automatic 24-fold reduction system directly connected to an elemental analyzer (EA; Hong et al. 2010a). The samples were sequentially combusted in the EA and the $\mathrm{CO}_{2}$ gases were transferred to the reduction system and trapped cryogenically in reduction tubes. Some 3-4 mg of Fe catalyst and hydrogen gas with a volume 2.1 times larger than that of $\mathrm{CO}_{2}$ was used for the reduction reactions at $600^{\circ} \mathrm{C}$. Typically, the reduction yields were $93 \%$ after a 3-hr reduction process. Around $1 \mathrm{mg}$ of graphite was obtained for each tree ring, and the graphite samples were pelletized for AMS measurements. ${ }^{14} \mathrm{C} /{ }^{12} \mathrm{C}$ ratios were measured using the 1MV AMS at KIGAM (Hong et al. 2010b). Each sample was measured 3 times for $1050 \mathrm{~s}$. The total counting time per tree-ring sample was $53 \mathrm{~min}$ and the total collected charge of 


\section{W Hong et al.}

${ }^{12} \mathrm{C}$ was $\sim 300-400 \mu \mathrm{C}$. To monitor the counting conditions, known samples (IAEA C7 and C8 reference materials) were measured every 25 tree-ring measurements.

\section{RESULTS AND DISCUSSION}

Results of the ${ }^{14} \mathrm{C}$ measurements of the annual tree-ring samples are presented in Table 2 with their dendrochronological ages. The ${ }^{14} \mathrm{C}$ concentration deviations ${ }^{14} \mathrm{C}$ of the tree rings grown on the Korean Peninsula during AD 1650-1850 were calculated by

$$
\Delta^{14} C=[p M C \cdot \exp \{(1950-y) / \lambda\}-1] \times 1000
$$

where $y$ is the year the ring was grown and $\lambda=\mathrm{t}_{1 / 2} / \ln (2)=8267$. $\mathrm{t}_{1 / 2}$ is the half-life of ${ }^{14} \mathrm{C}, 5730 \mathrm{yr}$. pMC was calculated by comparing the measured activity of a tree-ring sample to that of NIST oxalic acid after background subtraction, followed by $\delta^{13} \mathrm{C}$ correction following Stuiver and Polach (1977). ${ }^{13} \mathrm{C} /{ }^{12} \mathrm{C}$ ratios were measured by AMS, and the errors given in the Table 2 were evaluated by the statistical errors of the ${ }^{14} \mathrm{C}$ counts and ${ }^{12} \mathrm{C}$ and ${ }^{13} \mathrm{C}$ values of tree-ring samples, oxalic acids, and blank samples. Though the $\delta^{13} \mathrm{C}$ values of the $\mathrm{AD} 1657,1677,1682$, and 1728 samples were too high in terms of our $\delta^{13} \mathrm{C}$ criteria for terrestrial samples $\left(-30 \% \circ<\delta^{13} \mathrm{C} \leq-20 \%\right.$ ), their ages were consistent with the adjacent ages. The annual ${ }^{14} \mathrm{C}$ values of the tree rings measured are plotted in Figure 2 with the IntCal04 data. Because the IntCal04 data are the average values for every $10 \mathrm{yr}$ (Reimer et al. 2004), our tree-ring data were also averaged every $10 \mathrm{yr}$ to calculate the deviations in the Korean tree-ring data from the IntCal04 data. They are plotted every $5 \mathrm{yr}$ in Figure 2.

Table 2 Results of ${ }^{14} \mathrm{C}$ and dendrochronological age measurements of tree-ring samples grown in Korea from AD 1650 to 1850 . Ages (AD) were determined by the dendrochronological method, and $\delta^{13} \mathrm{C}$ values were measured by AMS.

\begin{tabular}{lllrrr}
\hline Lab code & Age (AD) & Year BP & \multicolumn{1}{l}{ pMC $(\%)$} & \multicolumn{1}{c}{$\Delta^{14} \mathrm{C}(\%)$} & \multicolumn{1}{c}{$\delta^{13} \mathrm{C}(\%)$} \\
\hline KGM-TWd091535 & 1650 & $190 \pm 30$ & $97.68 \pm 0.38$ & $12.93 \pm 3.80$ & $-23.83 \pm 0.16$ \\
KGM-TWd091534 & 1651 & $220 \pm 30$ & $97.31 \pm 0.38$ & $8.89 \pm 3.81$ & $-24.78 \pm 0.15$ \\
KGM-TWd091533 & 1652 & $269 \pm 30$ & $96.71 \pm 0.36$ & $2.60 \pm 3.60$ & $-21.39 \pm 3.40$ \\
KGM-TWd091532 & 1653 & $302 \pm 31$ & $96.31 \pm 0.37$ & $-1.67 \pm 3.66$ & $-22.95 \pm 3.81$ \\
KGM-TWd091531 & 1654 & $240 \pm 31$ & $97.06 \pm 0.38$ & $5.98 \pm 3.73$ & $-23.18 \pm 3.33$ \\
KGM-TWd091530 & 1655 & $254 \pm 32$ & $96.89 \pm 0.38$ & $4.10 \pm 3.80$ & $-23.55 \pm 3.24$ \\
KGM-TWd091529 & 1656 & $283 \pm 32$ & $96.54 \pm 0.38$ & $0.35 \pm 3.81$ & $-23.01 \pm 3.31$ \\
KGM-TWd091528 & 1657 & $323 \pm 31$ & $96.06 \pm 0.36$ & $-4.74 \pm 3.62$ & $-19.39 \pm 3.60$ \\
KGM-TWd091527 & 1658 & $275 \pm 30$ & $96.63 \pm 0.37$ & $1.04 \pm 3.63$ & $-21.63 \pm 1.36$ \\
KGM-TWd091526 & 1659 & $293 \pm 31$ & $96.42 \pm 0.37$ & $-1.26 \pm 3.70$ & $-23.29 \pm 0.27$ \\
KGM-TWd091525 & 1660 & $345 \pm 32$ & $95.79 \pm 0.38$ & $-7.90 \pm 3.80$ & $-22.95 \pm 0.66$ \\
KGM-TWd091524 & 1661 & $283 \pm 31$ & $96.54 \pm 0.38$ & $-0.25 \pm 3.74$ & $-21.53 \pm 0.44$ \\
KGM-TWd091523 & 1662 & $245 \pm 32$ & $96.99 \pm 0.39$ & $4.28 \pm 3.83$ & $-24.70 \pm 1.44$ \\
KGM-TWd091522 & 1663 & $293 \pm 32$ & $96.42 \pm 0.38$ & $-1.74 \pm 3.80$ & $-22.99 \pm 0.87$ \\
KGM-TWd091521 & 1664 & $255 \pm 31$ & $96.87 \pm 0.38$ & $2.80 \pm 3.73$ & $-21.72 \pm 0.97$ \\
KGM-TWd091520 & 1665 & $206 \pm 31$ & $97.47 \pm 0.38$ & $8.89 \pm 3.79$ & $-23.23 \pm 1.31$ \\
KGM-TWd091519 & 1666 & $199 \pm 30$ & $97.55 \pm 0.37$ & $9.59 \pm 3.65$ & $-24.01 \pm 0.62$ \\
KGM-TWd091518 & 1667 & $214 \pm 31$ & $97.37 \pm 0.37$ & $7.61 \pm 3.70$ & $-22.92 \pm 0.73$ \\
KGM-TWd091517 & 1668 & $198 \pm 31$ & $97.57 \pm 0.38$ & $9.56 \pm 3.78$ & $-20.65 \pm 1.05$ \\
KGM-TWd091516 & 1669 & $212 \pm 32$ & $97.40 \pm 0.39$ & $7.68 \pm 3.87$ & $-23.71 \pm 0.29$ \\
KGM-TWd091515 & 1670 & $206 \pm 32$ & $97.47 \pm 0.39$ & $8.28 \pm 3.89$ & $-25.15 \pm 0.86$ \\
KGM-TWd091514 & 1671 & $166 \pm 32$ & $97.95 \pm 0.39$ & $13.12 \pm 3.83$ & $-23.78 \pm 1.19$ \\
KGM-TWd091513 & 1672 & $174 \pm 32$ & $97.86 \pm 0.39$ & $12.07 \pm 3.85$ & $-23.40 \pm 1.01$ \\
KGM-TWd091512 & 1673 & $214 \pm 31$ & $97.37 \pm 0.37$ & $6.88 \pm 3.68$ & $-20.19 \pm 0.93$ \\
KGM-TWd091511 & 1674 & $170 \pm 32$ & $97.91 \pm 0.38$ & $12.34 \pm 3.82$ & $-23.48 \pm 2.11$ \\
KGM-TWd091510 & 1675 & $203 \pm 33$ & $97.51 \pm 0.40$ & $8.08 \pm 3.96$ & $-22.68 \pm 1.34$
\end{tabular}




\section{Regional Offset \& Variation in Korean Atmosphere}

Table 2 Results of ${ }^{14} \mathrm{C}$ and dendrochronological age measurements of tree-ring samples grown in Korea from $\mathrm{AD} 1650$ to 1850 . Ages (AD) were determined by the dendrochronological method, and $\delta^{13} \mathrm{C}$ values were measured by AMS. (Continued)

\begin{tabular}{|c|c|c|c|c|c|}
\hline Lab code & Age (AD) & Year BP & pMC (\%) & $\Delta^{14} \mathrm{C}$ (\%о) & $\delta^{13} \mathrm{C}(\%)$ \\
\hline KGM-TWd091509 & 1676 & $119 \pm 32$ & $98.53 \pm 0.39$ & $18.50 \pm 3.90$ & $-21.67 \pm 0.84$ \\
\hline KGM-TWd091508 & 1677 & $159 \pm 31$ & $98.04 \pm 0.38$ & $13.32 \pm 3.78$ & $-17.51 \pm 1.17$ \\
\hline KGM-TWd091507 & 1678 & $146 \pm 32$ & $98.20 \pm 0.40$ & $14.85 \pm 3.93$ & $-24.08 \pm 1.02$ \\
\hline KGM-TWd091506 & 1679 & $134 \pm 31$ & $98.35 \pm 0.38$ & $16.27 \pm 3.73$ & $-20.92 \pm 0.85$ \\
\hline KGM-TWd091505 & 1680 & $130 \pm 32$ & $98.39 \pm 0.39$ & $16.56 \pm 3.87$ & $-22.56 \pm 0.59$ \\
\hline KGM-TWd091504 & 1681 & $191 \pm 32$ & $97.65 \pm 0.39$ & $8.80 \pm 3.85$ & $-20.39 \pm 0.66$ \\
\hline KGM-TWd091503 & 1682 & $150 \pm 31$ & $98.15 \pm 0.38$ & $13.84 \pm 3.76$ & $-18.51 \pm 3.04$ \\
\hline KGM-TWd091502 & 1683 & $131 \pm 31$ & $98.39 \pm 0.38$ & $16.20 \pm 3.77$ & $-20.20 \pm 1.58$ \\
\hline KGM-TWd091501 & 1684 & $101 \pm 31$ & $98.75 \pm 0.39$ & $19.79 \pm 3.83$ & $-20.47 \pm 2.92$ \\
\hline KGM-TWd091500 & 1685 & $195 \pm 31$ & $97.60 \pm 0.38$ & $7.79 \pm 3.73$ & $-23.09 \pm 0.44$ \\
\hline KGM-TWd091499 & 1686 & $190 \pm 31$ & $97.66 \pm 0.38$ & $8.29 \pm 3.73$ & $-24.53 \pm 0.82$ \\
\hline KGM-TWd091498 & 1687 & $189 \pm 31$ & $97.67 \pm 0.38$ & $8.27 \pm 3.73$ & $-21.31 \pm 0.75$ \\
\hline KGM-TWd091497 & 1688 & $158 \pm 31$ & $98.05 \pm 0.38$ & $12.07 \pm 3.74$ & $-24.58 \pm 0.34$ \\
\hline KGM-TWd091496 & 1689 & $127 \pm 31$ & $98.43 \pm 0.38$ & $15.87 \pm 3.76$ & $-23.16 \pm 0.71$ \\
\hline KGM-TWd091495 & 1690 & $133 \pm 31$ & $98.36 \pm 0.38$ & $15.03 \pm 3.75$ & $-22.69 \pm 1.08$ \\
\hline KGM-TWd091494 & 1691 & $150 \pm 31$ & $98.16 \pm 0.38$ & $12.84 \pm 3.76$ & $-22.65 \pm 0.66$ \\
\hline KGM-TWd091493 & 1692 & $147 \pm 31$ & $98.19 \pm 0.38$ & $13.03 \pm 3.75$ & $-24.04 \pm 0.81$ \\
\hline KGM-TWd091492 & 1693 & $160 \pm 31$ & $98.03 \pm 0.38$ & $11.25 \pm 3.74$ & $-22.04 \pm 1.17$ \\
\hline KGM-TWd091491 & 1694 & $197 \pm 31$ & $97.57 \pm 0.38$ & $6.39 \pm 3.73$ & $-21.29 \pm 0.62$ \\
\hline KGM-TWd091490 & 1695 & $104 \pm 31$ & $98.71 \pm 0.38$ & $18.02 \pm 3.78$ & $-22.84 \pm 0.54$ \\
\hline KGM-TWd091489 & 1696 & $182 \pm 31$ & $97.75 \pm 0.38$ & $8.00 \pm 3.73$ & $-23.02 \pm 0.94$ \\
\hline KGM-TWd091488 & 1697 & $64 \pm 31$ & $99.20 \pm 0.38$ & $22.83 \pm 3.79$ & $-22.36 \pm 0.28$ \\
\hline KGM-TWd091487 & 1698 & $83 \pm 31$ & $98.97 \pm 0.38$ & $20.33 \pm 3.77$ & $-22.15 \pm 0.77$ \\
\hline KGM-TWd091486 & 1699 & $83 \pm 31$ & $98.97 \pm 0.38$ & $20.21 \pm 3.78$ & $-21.43 \pm 1.16$ \\
\hline KGM-TWd091485 & 1700 & $111 \pm 31$ & $98.63 \pm 0.38$ & $16.58 \pm 3.77$ & $-22.39 \pm 0.61$ \\
\hline KGM-TWd091484 & 1701 & $93 \pm 31$ & $98.85 \pm 0.38$ & $18.73 \pm 3.78$ & $-21.47 \pm 0.38$ \\
\hline KGM-TWd091483 & 1702 & $106 \pm 31$ & $98.69 \pm 0.38$ & $16.95 \pm 3.77$ & $-25.92 \pm 1.16$ \\
\hline KGM-TWd091482 & 1703 & $106 \pm 31$ & $98.69 \pm 0.38$ & $16.83 \pm 3.78$ & $-26.31 \pm 1.32$ \\
\hline KGM-TWd091481 & 1704 & $105 \pm 31$ & $98.70 \pm 0.38$ & $16.81 \pm 3.78$ & $-24.49 \pm 0.85$ \\
\hline KGM-TWd091480 & 1705 & $112 \pm 31$ & $98.62 \pm 0.38$ & $15.86 \pm 3.76$ & $-22.29 \pm 0.75$ \\
\hline KGM-TWd091479 & 1706 & $97 \pm 31$ & $98.80 \pm 0.38$ & $17.60 \pm 3.78$ & $-22.92 \pm 0.90$ \\
\hline KGM-TWd091478 & 1707 & $76 \pm 31$ & $99.06 \pm 0.38$ & $20.15 \pm 3.78$ & $-25.12 \pm 0.92$ \\
\hline KGM-TWd091477 & 1708 & $136 \pm 31$ & $98.32 \pm 0.38$ & $12.41 \pm 3.75$ & $-23.24 \pm 0.83$ \\
\hline KGM-TWd091476 & 1709 & $-26 \pm 34$ & $100.32 \pm 0.43$ & $32.88 \pm 4.25$ & $-21.27 \pm 0.08$ \\
\hline KGM-TWd091475 & 1710 & $-112 \pm 34$ & $101.40 \pm 0.43$ & $43.87 \pm 4.26$ & $-20.25 \pm 0.13$ \\
\hline KGM-TWd091474 & 1711 & $-62 \pm 34$ & $100.77 \pm 0.42$ & $37.26 \pm 4.19$ & $-23.44 \pm 0.13$ \\
\hline KGM-TWd091473 & 1712 & $-6 \pm 34$ & $100.07 \pm 0.42$ & $29.93 \pm 4.18$ & $-23.97 \pm 0.44$ \\
\hline KGM-TWd091472 & 1713 & $173 \pm 35$ & $97.87 \pm 0.42$ & $7.16 \pm 4.17$ & $-24.20 \pm 0.39$ \\
\hline KGM-TWd091471 & 1714 & $-21 \pm 34$ & $100.27 \pm 0.43$ & $31.74 \pm 4.26$ & $-22.68 \pm 0.10$ \\
\hline KGM-TWd091470 & 1715 & $31 \pm 30$ & $99.61 \pm 0.38$ & $24.82 \pm 3.73$ & $-23.26 \pm 2.28$ \\
\hline KGM-TWd091469 & 1716 & $108 \pm 31$ & $98.67 \pm 0.38$ & $15.03 \pm 3.73$ & $-25.00 \pm 2.27$ \\
\hline KGM-TWd091468 & 1717 & $88 \pm 31$ & $98.91 \pm 0.38$ & $17.37 \pm 3.78$ & $-22.51 \pm 0.55$ \\
\hline KGM-TWd091467 & 1718 & $43 \pm 31$ & $99.47 \pm 0.38$ & $23.01 \pm 3.79$ & $-21.52 \pm 0.81$ \\
\hline KGM-TWd091466 & 1719 & $80 \pm 31$ & $99.01 \pm 0.38$ & $18.16 \pm 3.79$ & $-23.95 \pm 1.11$ \\
\hline KGM-TWd091465 & 1720 & $116 \pm 31$ & $98.57 \pm 0.38$ & $13.51 \pm 3.75$ & $-23.27 \pm 0.64$ \\
\hline KGM-TWd091464 & 1721 & $114 \pm 31$ & $98.59 \pm 0.38$ & $13.59 \pm 3.77$ & $-22.45 \pm 1.27$ \\
\hline KGM-TWd091463-1 & 1722 & $146 \pm 32$ & $98.20 \pm 0.39$ & $9.46 \pm 3.83$ & $-21.44 \pm 0.50$ \\
\hline KGM-TWd091462-1 & 1723 & $185 \pm 32$ & $97.73 \pm 0.38$ & $4.51 \pm 3.80$ & $-21.79 \pm 0.41$ \\
\hline KGM-TWd091461-1 & 1724 & $180 \pm 33$ & $97.79 \pm 0.39$ & $5.00 \pm 3.92$ & $-23.22 \pm 0.55$ \\
\hline KGM-TWd091460 & 1725 & $84 \pm 31$ & $98.97 \pm 0.38$ & $17.01 \pm 3.74$ & $-23.96 \pm 2.16$ \\
\hline KGM-TWd091459 & 1726 & $165 \pm 31$ & $97.97 \pm 0.38$ & $6.61 \pm 3.74$ & $-22.84 \pm 1.99$ \\
\hline KGM-TWd091458 & 1727 & $88 \pm 31$ & $98.91 \pm 0.38$ & $16.14 \pm 3.74$ & $-24.36 \pm 3.18$ \\
\hline KGM-TWd091457 & 1728 & $221 \pm 31$ & $97.29 \pm 0.37$ & $-0.62 \pm 3.70$ & $-19.91 \pm 2.95$ \\
\hline
\end{tabular}


W Hong et al.

Table 2 Results of ${ }^{14} \mathrm{C}$ and dendrochronological age measurements of tree-ring samples grown in Korea from $\mathrm{AD} 1650$ to 1850 . Ages (AD) were determined by the dendrochronological method, and $\delta^{13} \mathrm{C}$ values were measured by AMS. (Continued)

\begin{tabular}{|c|c|c|c|c|c|}
\hline Lab code & Age (AD) & Year BP & pMC (\%) & $\Delta^{14} \mathrm{C}(\%)$ & $\delta^{13} \mathrm{C}(\%)$ \\
\hline KGM-TWd091456 & 1729 & $123 \pm 31$ & $98.48 \pm 0.38$ & $11.48 \pm 3.80$ & $-21.66 \pm 0.93$ \\
\hline KGM-TWd091455 & 1730 & $143 \pm 31$ & $98.24 \pm 0.38$ & $8.89 \pm 3.72$ & $-21.26 \pm 0.84$ \\
\hline KGM-TWd091454 & 1731 & $60 \pm 32$ & $99.25 \pm 0.39$ & $19.14 \pm 3.88$ & $-24.17 \pm 0.87$ \\
\hline KGM-TWd091453 & 1732 & $80 \pm 31$ & $99.00 \pm 0.38$ & $16.45 \pm 3.75$ & $-23.10 \pm 0.55$ \\
\hline KGM-TWd091452 & 1733 & $121 \pm 31$ & $98.51 \pm 0.39$ & $11.30 \pm 3.82$ & $-23.03 \pm 0.29$ \\
\hline KGM-TWd091451 & 1734 & $112 \pm 31$ & $98.62 \pm 0.38$ & $12.31 \pm 3.79$ & $-22.26 \pm 0.69$ \\
\hline KGM-TWd091450 & 1735 & $173 \pm 32$ & $97.87 \pm 0.38$ & $4.49 \pm 3.80$ & $-22.78 \pm 0.76$ \\
\hline KGM-TWd091449 & 1736 & $201 \pm 30$ & $97.53 \pm 0.37$ & $0.88 \pm 3.66$ & $-21.26 \pm 0.61$ \\
\hline KGM-TWd091448 & 1737 & $200 \pm 32$ & $97.55 \pm 0.38$ & $0.96 \pm 3.80$ & $-23.02 \pm 0.75$ \\
\hline KGM-TWd091447 & 1738 & $222 \pm 30$ & $97.28 \pm 0.37$ & $-1.93 \pm 3.65$ & $-21.11 \pm 0.49$ \\
\hline KGM-TWd091446 & 1739 & $191 \pm 31$ & $97.65 \pm 0.37$ & $1.74 \pm 3.69$ & $-21.87 \pm 0.20$ \\
\hline KGM-TWd091445 & 1740 & $224 \pm 31$ & $97.24 \pm 0.37$ & $-2.58 \pm 3.68$ & $-22.83 \pm 0.40$ \\
\hline KGM-TWd091444 & 1741 & $230 \pm 31$ & $97.18 \pm 0.37$ & $-3.32 \pm 3.66$ & $-23.34 \pm 0.57$ \\
\hline KGM-TWd091443 & 1742 & $222 \pm 31$ & $97.28 \pm 0.37$ & $-2.41 \pm 3.69$ & $-20.87 \pm 0.30$ \\
\hline KGM-TWd091442 & 1743 & $181 \pm 33$ & $97.78 \pm 0.40$ & $2.59 \pm 3.95$ & $-25.29 \pm 0.34$ \\
\hline KGM-TWd091441 & 1744 & $220 \pm 31$ & $97.30 \pm 0.37$ & $-2.45 \pm 3.66$ & $-22.05 \pm 0.24$ \\
\hline KGM-TWd091440 & 1745 & $191 \pm 32$ & $97.66 \pm 0.39$ & $1.12 \pm 3.89$ & $-24.32 \pm 0.91$ \\
\hline KGM-TWd091439 & 1746 & $200 \pm 33$ & $97.55 \pm 0.40$ & $-0.13 \pm 3.94$ & $-24.29 \pm 0.37$ \\
\hline KGM-TWd091438 & 1747 & $232 \pm 31$ & $97.15 \pm 0.38$ & $-4.35 \pm 3.77$ & $-23.04 \pm 0.35$ \\
\hline KGM-TWd091437 & 1748 & $201 \pm 30$ & $97.53 \pm 0.37$ & $-0.58 \pm 3.64$ & $-22.28 \pm 0.34$ \\
\hline KGM-TWd091436 & 1749 & $196 \pm 30$ & $97.59 \pm 0.37$ & $-0.08 \pm 3.65$ & $-21.08 \pm 0.68$ \\
\hline KGM-TCe110001 & 1750 & $191 \pm 30$ & $97.65 \pm 0.37$ & $0.41 \pm 3.63$ & $-22.20 \pm 0.80$ \\
\hline KGM-TCe110002 & 1751 & $223 \pm 41$ & $97.26 \pm 0.50$ & $-3.70 \pm 4.93$ & $-22.57 \pm 0.38$ \\
\hline KGM-TCe110003 & 1752 & $200 \pm 40$ & $97.53 \pm 0.49$ & $-1.06 \pm 4.82$ & $-23.57 \pm 0.16$ \\
\hline KGM-TCe110004 & 1753 & $270 \pm 41$ & $96.70 \pm 0.49$ & $-9.68 \pm 4.91$ & $-23.69 \pm 0.14$ \\
\hline KGM-TCe110005 & 1754 & $255 \pm 40$ & $96.87 \pm 0.48$ & $-8.06 \pm 4.73$ & $-23.30 \pm 0.10$ \\
\hline KGM-TCe110006 & 1755 & $266 \pm 41$ & $96.74 \pm 0.49$ & $-9.51 \pm 4.91$ & $-24.89 \pm 0.26$ \\
\hline KGM-TCe110007 & 1756 & $270 \pm 40$ & $96.69 \pm 0.48$ & $-10.14 \pm 4.73$ & $-22.34 \pm 0.21$ \\
\hline KGM-TCe110008 & 1757 & $247 \pm 41$ & $96.97 \pm 0.49$ & $-7.40 \pm 4.84$ & $-23.37 \pm 0.20$ \\
\hline KGM-TCe110009 & 1758 & $189 \pm 41$ & $97.68 \pm 0.50$ & $-0.25 \pm 4.99$ & $-22.81 \pm 0.17$ \\
\hline KGM-TCe110010 & 1759 & $171 \pm 41$ & $97.89 \pm 0.50$ & $1.78 \pm 4.97$ & $-22.59 \pm 0.38$ \\
\hline KGM-TCe110011 & 1760 & $195 \pm 41$ & $97.60 \pm 0.50$ & $-1.31 \pm 4.94$ & $-21.93 \pm 0.39$ \\
\hline KGM-TCe110012 & 1761 & $272 \pm 40$ & $96.68 \pm 0.48$ & $-10.84 \pm 4.80$ & $-20.95 \pm 0.41$ \\
\hline KGM-TCe110013 & 1762 & $203 \pm 31$ & $97.51 \pm 0.38$ & $-2.47 \pm 3.76$ & $-23.43 \pm 0.37$ \\
\hline KGM-TCe110014 & 1763 & $193 \pm 31$ & $97.63 \pm 0.38$ & $-1.36 \pm 3.74$ & $-22.92 \pm 0.49$ \\
\hline KGM-TCe110015 & 1764 & $179 \pm 31$ & $97.79 \pm 0.38$ & $0.15 \pm 3.79$ & $-22.51 \pm 0.38$ \\
\hline KGM-TCe110016 & 1765 & $169 \pm 32$ & $97.92 \pm 0.38$ & $1.36 \pm 3.82$ & $-24.24 \pm 0.37$ \\
\hline KGM-TCe110017 & 1766 & $180 \pm 31$ & $97.79 \pm 0.37$ & $-0.09 \pm 3.70$ & $-22.66 \pm 1.45$ \\
\hline KGM-TCe110018 & 1767 & $226 \pm 32$ & $97.23 \pm 0.39$ & $-5.94 \pm 3.86$ & $-22.78 \pm 1.52$ \\
\hline KGM-TCe110019 & 1768 & $207 \pm 31$ & $97.45 \pm 0.38$ & $-3.81 \pm 3.77$ & $-21.89 \pm 1.19$ \\
\hline KGM-TCe110020 & 1769 & $146 \pm 31$ & $98.20 \pm 0.38$ & $3.74 \pm 3.77$ & $-23.95 \pm 1.33$ \\
\hline KGM-TCe110021 & 1770 & $171 \pm 31$ & $97.90 \pm 0.38$ & $0.55 \pm 3.80$ & $-23.16 \pm 1.06$ \\
\hline KGM-TCe110022 & 1771 & $155 \pm 32$ & $98.09 \pm 0.39$ & $2.37 \pm 3.84$ & $-23.94 \pm 1.82$ \\
\hline KGM-TCe110023 & 1772 & $198 \pm 33$ & $97.57 \pm 0.40$ & $-3.06 \pm 4.00$ & $-23.58 \pm 0.32$ \\
\hline KGM-TCe110024 & 1773 & $167 \pm 31$ & $97.94 \pm 0.38$ & $0.60 \pm 3.72$ & $-22.30 \pm 0.17$ \\
\hline KGM-TCe110025 & 1774 & $218 \pm 31$ & $97.32 \pm 0.38$ & $-5.86 \pm 3.75$ & $-20.95 \pm 0.65$ \\
\hline KGM-TCe110026 & 1775 & $156 \pm 31$ & $98.08 \pm 0.38$ & $1.78 \pm 3.77$ & $-22.48 \pm 0.39$ \\
\hline KGM-TCe110027 & 1776 & $194 \pm 31$ & $97.61 \pm 0.38$ & $-3.14 \pm 3.73$ & $-22.24 \pm 0.21$ \\
\hline KGM-TCe110028 & 1777 & $192 \pm 31$ & $97.64 \pm 0.37$ & $-2.95 \pm 3.69$ & $-20.96 \pm 0.58$ \\
\hline KGM-TCe110029 & 1778 & $146 \pm 32$ & $98.20 \pm 0.39$ & $2.65 \pm 3.86$ & $-21.19 \pm 0.44$ \\
\hline KGM-TCe110030 & 1779 & $250 \pm 31$ & $96.94 \pm 0.38$ & $-10.34 \pm 3.74$ & $-21.90 \pm 0.42$ \\
\hline KGM-TCe110031 & 1780 & $189 \pm 31$ & $97.68 \pm 0.37$ & $-2.91 \pm 3.72$ & $-20.86 \pm 0.58$ \\
\hline KGM-TCe110032 & 1781 & $184 \pm 32$ & $97.74 \pm 0.39$ & $-2.41 \pm 3.91$ & $-23.30 \pm 0.33$ \\
\hline
\end{tabular}




\section{Regional Offset \& Variation in Korean Atmosphere}

Table 2 Results of ${ }^{14} \mathrm{C}$ and dendrochronological age measurements of tree-ring samples grown in Korea from $\mathrm{AD} 1650$ to 1850 . Ages (AD) were determined by the dendrochronological method, and $\delta^{13} \mathrm{C}$ values were measured by AMS. (Continued)

\begin{tabular}{|c|c|c|c|c|c|}
\hline Lab code & Age (AD) & Year BP & pMC (\%) & $\Delta^{14} \mathrm{C}$ (\%о) & $\delta^{13} \mathrm{C}(\%)$ \\
\hline KGM-TCe110033 & 1782 & $209 \pm 32$ & $97.43 \pm 0.39$ & $-5.70 \pm 3.89$ & $-21.13 \pm 0.16$ \\
\hline KGM-TCe110034 & 1783 & $250 \pm 32$ & $96.93 \pm 0.39$ & $-10.92 \pm 3.88$ & $-22.18 \pm 0.21$ \\
\hline KGM-TCe110035 & 1784 & $194 \pm 32$ & $97.62 \pm 0.38$ & $-4.00 \pm 3.80$ & $-22.20 \pm 0.15$ \\
\hline KGM-TCe110036 & 1785 & $124 \pm 29$ & $98.46 \pm 0.35$ & $4.45 \pm 3.51$ & $-23.33 \pm 0.38$ \\
\hline KGM-TCe110037 & 1786 & $197 \pm 28$ & $97.57 \pm 0.34$ & $-4.75 \pm 3.35$ & $-23.42 \pm 0.63$ \\
\hline KGM-TCe110038 & 1787 & $121 \pm 27$ & $98.50 \pm 0.33$ & $4.61 \pm 3.27$ & $-22.17 \pm 0.11$ \\
\hline KGM-TCe110039 & 1788 & $114 \pm 27$ & $98.59 \pm 0.33$ & $5.41 \pm 3.26$ & $-21.88 \pm 0.19$ \\
\hline KGM-TCe110040 & 1789 & $56 \pm 27$ & $99.31 \pm 0.34$ & $12.63 \pm 3.35$ & $-23.43 \pm 0.41$ \\
\hline KGM-TCe110041 & 1790 & $194 \pm 28$ & $97.61 \pm 0.34$ & $-4.82 \pm 3.36$ & $-22.77 \pm 0.40$ \\
\hline KGM-TCe110042 & 1791 & $175 \pm 27$ & $97.85 \pm 0.33$ & $-2.50 \pm 3.31$ & $-22.95 \pm 0.35$ \\
\hline KGM-TCe110043 & 1792 & $213 \pm 31$ & $97.38 \pm 0.38$ & $-7.41 \pm 3.74$ & $-22.99 \pm 0.53$ \\
\hline KGM-TCe110044 & 1793 & $282 \pm 27$ & $96.55 \pm 0.32$ & $-15.99 \pm 3.22$ & $-22.55 \pm 0.14$ \\
\hline KGM-TCe110045 & 1794 & $265 \pm 27$ & $96.75 \pm 0.33$ & $-14.07 \pm 3.24$ & $-21.53 \pm 0.56$ \\
\hline KGM-TCe110046 & 1795 & $216 \pm 28$ & $97.35 \pm 0.34$ & $-8.08 \pm 3.41$ & $-23.23 \pm 0.40$ \\
\hline KGM-TCe110047 & 1796 & $234 \pm 30$ & $97.12 \pm 0.36$ & $-10.54 \pm 3.56$ & $-23.64 \pm 0.59$ \\
\hline KGM-TCe110048 & 1797 & $216 \pm 28$ & $97.35 \pm 0.34$ & $-8.32 \pm 3.39$ & $-22.20 \pm 0.29$ \\
\hline KGM-TCe110049 & 1798 & $172 \pm 28$ & $97.88 \pm 0.35$ & $-3.04 \pm 3.44$ & $-22.26 \pm 0.52$ \\
\hline KGM-TCe110050 & 1799 & $149 \pm 28$ & $98.16 \pm 0.34$ & $-0.31 \pm 3.35$ & $-21.80 \pm 0.25$ \\
\hline KGM-TCe110051 & 1800 & $135 \pm 29$ & $98.33 \pm 0.35$ & $1.30 \pm 3.48$ & $-21.93 \pm 0.19$ \\
\hline KGM-TCe110052 & 1801 & $166 \pm 28$ & $97.95 \pm 0.34$ & $-2.69 \pm 3.38$ & $-21.59 \pm 0.22$ \\
\hline KGM-TCe110053 & 1802 & $167 \pm 28$ & $97.95 \pm 0.34$ & $-2.81 \pm 3.41$ & $-22.64 \pm 0.30$ \\
\hline KGM-TCe110054 & 1803 & $181 \pm 27$ & $97.77 \pm 0.33$ & $-4.76 \pm 3.27$ & $-21.90 \pm 0.18$ \\
\hline KGM-TCe110055 & 1804 & $149 \pm 28$ & $98.17 \pm 0.35$ & $-0.81 \pm 3.43$ & $-23.69 \pm 0.31$ \\
\hline KGM-TCe110056 & 1805 & $157 \pm 28$ & $98.07 \pm 0.34$ & $-1.95 \pm 3.36$ & $-22.07 \pm 0.44$ \\
\hline KGM-TCe110057 & 1806 & $155 \pm 27$ & $98.08 \pm 0.33$ & $-1.97 \pm 3.32$ & $-22.49 \pm 0.21$ \\
\hline KGM-TCe110058 & 1807 & $147 \pm 28$ & $98.18 \pm 0.34$ & $-1.07 \pm 3.33$ & $-22.54 \pm 0.27$ \\
\hline KGM-TCe110059 & 1808 & $127 \pm 28$ & $98.44 \pm 0.34$ & $1.45 \pm 3.36$ & $-22.73 \pm 0.38$ \\
\hline KGM-TCe110060 & 1809 & $209 \pm 28$ & $97.44 \pm 0.34$ & $-8.84 \pm 3.38$ & $-23.68 \pm 0.17$ \\
\hline KGM-TCe110061 & 1810 & $175 \pm 28$ & $97.85 \pm 0.34$ & $-4.79 \pm 3.40$ & $-23.99 \pm 0.34$ \\
\hline KGM-TCe110062 & 1811 & $183 \pm 28$ & $97.75 \pm 0.34$ & $-5.93 \pm 3.33$ & $-21.86 \pm 0.49$ \\
\hline KGM-TCe110063 & 1812 & $81 \pm 31$ & $99.00 \pm 0.38$ & $6.66 \pm 3.78$ & $-22.95 \pm 0.37$ \\
\hline KGM-TCe110064 & 1813 & $182 \pm 31$ & $97.76 \pm 0.37$ & $-6.06 \pm 3.69$ & $-21.60 \pm 0.33$ \\
\hline KGM-TCe110065 & 1814 & $50 \pm 30$ & $99.37 \pm 0.38$ & $10.18 \pm 3.74$ & $-22.24 \pm 0.36$ \\
\hline KGM-TCe110066 & 1815 & $79 \pm 30$ & $99.02 \pm 0.37$ & $6.50 \pm 3.70$ & $-21.02 \pm 0.20$ \\
\hline KGM-TCe110067 & 1816 & $93 \pm 30$ & $98.85 \pm 0.37$ & $4.65 \pm 3.69$ & $-21.17 \pm 0.61$ \\
\hline KGM-TCe110068 & 1817 & $71 \pm 30$ & $99.12 \pm 0.37$ & $7.28 \pm 3.71$ & $-23.20 \pm 0.36$ \\
\hline KGM-TCe110069 & 1818 & $118 \pm 31$ & $98.54 \pm 0.38$ & $1.26 \pm 3.72$ & $-22.79 \pm 0.45$ \\
\hline KGM-TCe110070 & 1819 & $54 \pm 30$ & $99.33 \pm 0.37$ & $9.17 \pm 3.72$ & $-22.23 \pm 0.36$ \\
\hline KGM-TCe110071 & 1820 & $80 \pm 31$ & $99.01 \pm 0.38$ & $5.79 \pm 3.76$ & $-24.42 \pm 0.57$ \\
\hline KGM-TCe110072 & 1821 & $46 \pm 30$ & $99.43 \pm 0.37$ & $9.94 \pm 3.71$ & $-22.38 \pm 0.66$ \\
\hline KGM-TCe110073 & 1822 & $63 \pm 31$ & $99.22 \pm 0.38$ & $7.68 \pm 3.74$ & $-24.37 \pm 0.70$ \\
\hline KGM-TCe110074 & 1823 & $86 \pm 30$ & $98.94 \pm 0.37$ & $4.72 \pm 3.69$ & $-20.98 \pm 0.58$ \\
\hline KGM-TCe110075 & 1824 & $86 \pm 30$ & $98.94 \pm 0.37$ & $4.60 \pm 3.69$ & $-23.36 \pm 0.14$ \\
\hline KGM-TCe110076 & 1825 & $51 \pm 31$ & $99.36 \pm 0.38$ & $8.74 \pm 3.76$ & $-23.41 \pm 0.28$ \\
\hline KGM-TCe110077 & 1826 & $101 \pm 31$ & $98.75 \pm 0.38$ & $2.42 \pm 3.74$ & $-23.91 \pm 0.90$ \\
\hline KGM-TCe110078 & 1827 & $126 \pm 31$ & $98.44 \pm 0.38$ & $-0.84 \pm 3.74$ & $-25.85 \pm 1.04$ \\
\hline KGM-TCe110079 & 1828 & $60 \pm 30$ & $99.26 \pm 0.37$ & $7.36 \pm 3.72$ & $-22.31 \pm 0.63$ \\
\hline KGM-TCe110080 & 1829 & $105 \pm 30$ & $98.70 \pm 0.37$ & $1.55 \pm 3.69$ & $-22.54 \pm 0.38$ \\
\hline KGM-TCe110081 & 1830 & $84 \pm 31$ & $98.96 \pm 0.38$ & $4.07 \pm 3.77$ & $-27.41 \pm 0.20$ \\
\hline KGM-TCe110082 & 1831 & $70 \pm 31$ & $99.13 \pm 0.38$ & $5.67 \pm 3.75$ & $-23.85 \pm 0.39$ \\
\hline KGM-TCe110083 & 1832 & $96 \pm 31$ & $98.81 \pm 0.38$ & $2.30 \pm 3.82$ & $-21.79 \pm 0.63$ \\
\hline KGM-TCe110084 & 1833 & $106 \pm 30$ & $98.69 \pm 0.37$ & $0.97 \pm 3.68$ & $-22.00 \pm 1.43$ \\
\hline KGM-TCe110085 & 1834 & $166 \pm 31$ & $97.96 \pm 0.37$ & $-6.56 \pm 3.70$ & $-23.25 \pm 1.17$ \\
\hline
\end{tabular}




\section{W Hong et al.}

Table 2 Results of ${ }^{14} \mathrm{C}$ and dendrochronological age measurements of tree-ring samples grown in Korea from $\mathrm{AD} 1650$ to 1850 . Ages (AD) were determined by the dendrochronological method, and $\delta^{13} \mathrm{C}$ values were measured by AMS. (Continued)

\begin{tabular}{llrrrr}
\hline Lab code & Age $(\mathrm{AD})$ & \multicolumn{1}{c}{ Year BP } & \multicolumn{1}{c}{ pMC $(\%)$} & \multicolumn{1}{c}{$\Delta^{14} \mathrm{C}(\%$ o $)$} & \multicolumn{1}{c}{$\delta^{13} \mathrm{C}(\%$ о) } \\
\hline KGM-TCe110086 & 1835 & $169 \pm 30$ & $97.92 \pm 0.37$ & $-7.08 \pm 3.66$ & $-22.29 \pm 1.57$ \\
KGM-TCe110087 & 1836 & $101 \pm 31$ & $98.75 \pm 0.38$ & $1.21 \pm 3.77$ & $-22.50 \pm 1.26$ \\
KGM-TCe110088 & 1837 & $79 \pm 30$ & $99.02 \pm 0.37$ & $3.83 \pm 3.69$ & $-23.64 \pm 0.61$ \\
KGM-TCe110089 & 1838 & $89 \pm 30$ & $98.89 \pm 0.37$ & $2.39 \pm 3.69$ & $-23.16 \pm 0.20$ \\
KGM-TCe110090 & 1839 & $118 \pm 30$ & $98.54 \pm 0.37$ & $-1.28 \pm 3.64$ & $-23.12 \pm 0.41$ \\
KGM-TCe110091 & 1840 & $185 \pm 31$ & $97.72 \pm 0.37$ & $-9.71 \pm 3.68$ & $-25.31 \pm 0.98$ \\
KGM-TCe110092 & 1841 & $180 \pm 29$ & $97.78 \pm 0.36$ & $-9.22 \pm 3.53$ & $-23.21 \pm 0.16$ \\
KGM-TCe110093 & 1842 & $180 \pm 31$ & $97.78 \pm 0.38$ & $-9.34 \pm 3.73$ & $-25.06 \pm 0.69$ \\
KGM-TCe110094 & 1843 & $184 \pm 30$ & $97.74 \pm 0.36$ & $-9.87 \pm 3.60$ & $-24.53 \pm 0.36$ \\
KGM-TCe110095 & 1844 & $121 \pm 30$ & $98.51 \pm 0.37$ & $-2.19 \pm 3.62$ & $-25.00 \pm 0.20$ \\
KGM-TCe110096 & 1845 & $136 \pm 30$ & $98.32 \pm 0.37$ & $-4.23 \pm 3.65$ & $-24.55 \pm 0.25$ \\
KGM-TCe110097 & 1846 & $139 \pm 30$ & $98.29 \pm 0.36$ & $-4.66 \pm 3.59$ & $-24.40 \pm 0.20$ \\
KGM-TCe110098 & 1847 & $70 \pm 29$ & $99.13 \pm 0.36$ & $3.73 \pm 3.58$ & $-24.16 \pm 0.80$ \\
KGM-TCe110099 & 1848 & $135 \pm 29$ & $98.33 \pm 0.36$ & $-4.49 \pm 3.55$ & $-23.18 \pm 1.14$ \\
KGM-TCe110100 & 1849 & $126 \pm 29$ & $98.45 \pm 0.36$ & $-3.40 \pm 3.55$ & $-22.57 \pm 0.98$ \\
KGM-TCe110101 & 1850 & $131 \pm 30$ & $98.38 \pm 0.37$ & $-4.23 \pm 3.66$ & $-22.80 \pm 0.18$ \\
\hline
\end{tabular}

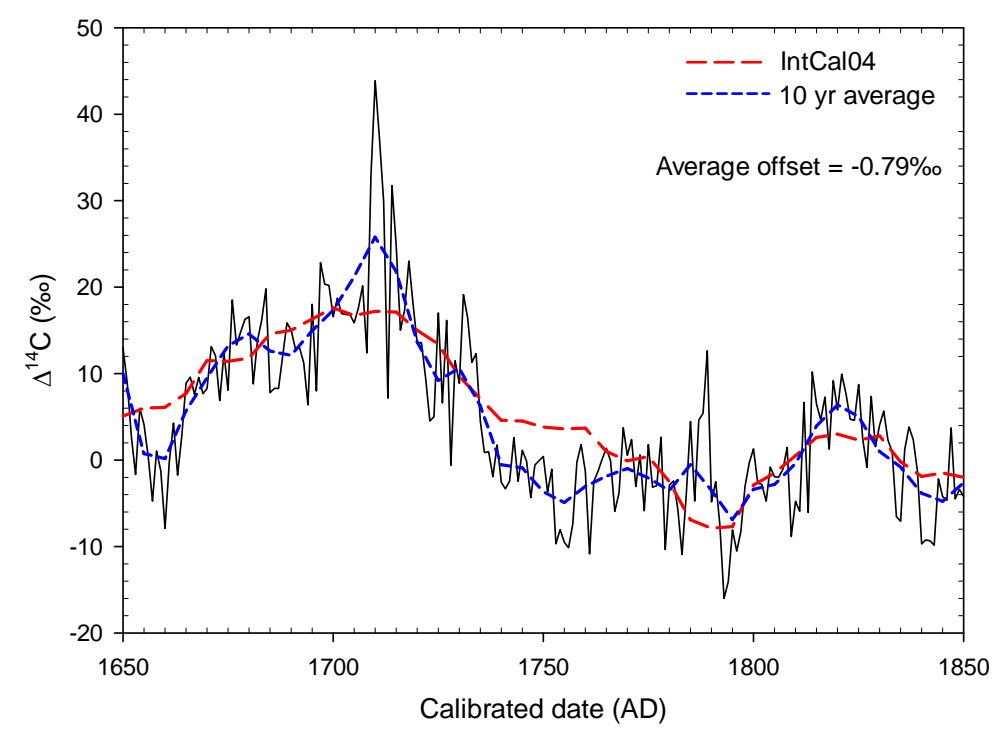

Figure 2 Variation curve (solid line) of ${ }^{14} \mathrm{C}$ concentration in the Korean atmosphere from AD 1650 to 1850. The dashed line represents IntCal04 data and the dotted line, the Korean measurements smoothed by averaging every $10 \mathrm{yr}$. The average offset is $-0.79 \%$.

The average deviation of the ${ }^{14} \mathrm{C}$ concentrations of Korean tree rings from the IntCal data for $\mathrm{AD} 1650$ to 1850 was calculated as $-0.79 \pm 3.81 \%$, which is a much smaller value than the statistical error. Hence, the deviation can be ignored when IntCal data are used for calibration of the ${ }^{14} \mathrm{C}$ ages of Korean samples. Our previous work showed that the average deviation of ${ }^{14} \mathrm{C}$ concentration of Korean tree rings from AD 1250 to $1650,-2.13 \pm 4.32 \%$, was a much larger value than $-0.79 \%$ (Hong et al. 2013). A remarkable change between the offsets of the 2 age ranges was observed. The low ${ }^{14} \mathrm{C}$ concentration in the east Asian atmosphere is thought to be due to the release of ${ }^{14} \mathrm{C}$-depleted $\mathrm{CO}_{2}$ from the Kuroshio Current in the northern Pacific Ocean, which is a warm 


\section{Regional Offset \& Variation in Korean Atmosphere}

current starting near the Taiwanese islands and passing through the straits between the Korean Peninsula and Japan, i.e. the Korea Strait. During the summer season, when most tree growth occurs, southeasters containing the ${ }^{14} \mathrm{C}$-depleted $\mathrm{CO}_{2}$ affect this country dominantly. Thus, the ${ }^{14} \mathrm{C}$ concentrations in the Korean trees are strongly affected by the Kuroshio. It is known that the temperature during the Little Ice Age (LIA) from AD 1550 to 1850 was lower by at least $1{ }^{\circ} \mathrm{C}$ than the temperature before and after (Mann 2002). The Maunder minimum (AD 1650-1700), a wellknown period of minimum solar activity (Eddy 1976), is suggested as a cause for the cold period. The LIA includes the age range of tree rings used in this work. The release rate of $\mathrm{CO}_{2}$ from the northern Pacific Ocean increases along with the temperature. The small offset of ${ }^{14} \mathrm{C}$ concentration of Korean trees from IntCal from AD 1650 to 1850 can be interpreted by understanding that the release rate of ${ }^{14} \mathrm{C}$-depleted $\mathrm{CO}_{2}$ from the Kuroshio Current decreased during the LIA such that the migration of ${ }^{14} \mathrm{C}$-depleted $\mathrm{CO}_{2}$ to the Korean atmosphere also decreased during this period. Also, a cold climate could make the intensity of the warm monsoon from the south small, while the influence of the cold continental high pressure from the north to the Korean climate could be relatively large. This could be another reason of the small offset of the period.

The relationship between the ${ }^{14} \mathrm{C}$ ages of the Korean tree rings and the dendrochronological ages is plotted in Figure 3 as a comparison to the IntCal04 calibration curve. The ${ }^{14} \mathrm{C}$ ages of the Korean tree rings around $\mathrm{AD} 1710$ and 1787 deviate from those in the IntCal04 data. The average ${ }^{14} \mathrm{C}$ offset from IntCal in these periods is $+5.93 \%$ o $\left(-47{ }^{14} \mathrm{C}\right.$ yr) around $\mathrm{AD} 1710$ and $+3.24 \%$ \% $\left(-25{ }^{14} \mathrm{C}\right.$ yr $)$ around $\mathrm{AD} 1787$, which are larger by $6.72 \%$ and $4.03 \%$, respectively, than the average of the entire period. Because the 2 periods are included in both timespans of TMJS017A (AD 1619-1804) and TMJS079A (AD 1699-1833), the ${ }^{14} \mathrm{C}$ concentrations during the periods will be cross-checked. A proper correction may then be necessary when the ages of samples around AD 1710 and 1787 are calibrated.

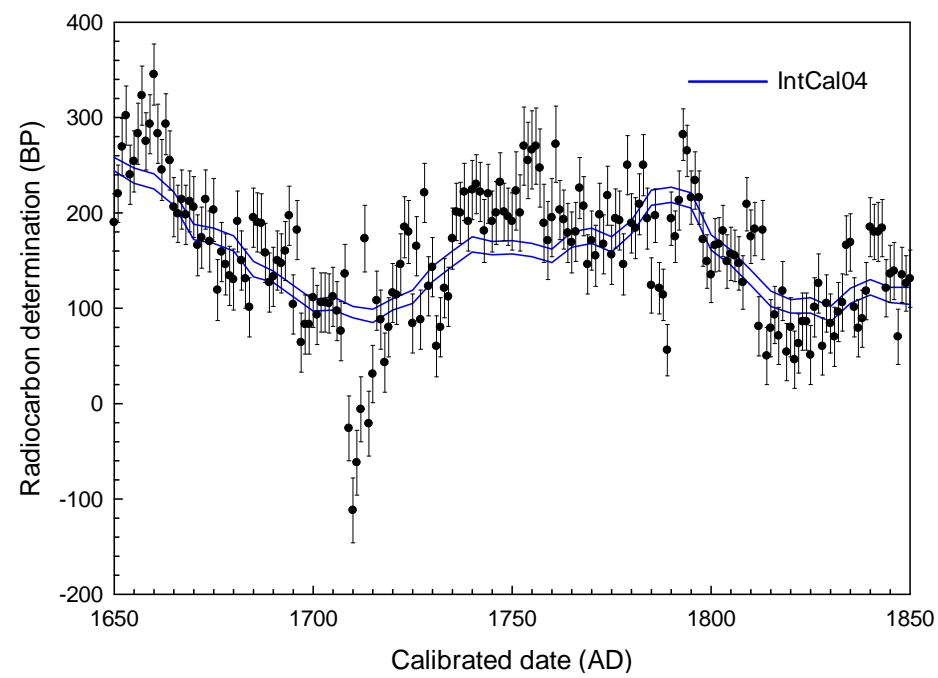

Figure 3 Comparison of ${ }^{14} \mathrm{C}$ ages of Korean tree rings grown AD $1650-1850$ and their dendrochronological ages with the calibration curve of IntCal04 (solid line).

\section{CONCLUSION}

Tree rings with ages spanning AD 1650 to 1850 were measured to verify the consistency of the IntCal data with data from local samples of Korea. Three samples were collected from an historic 


\section{W Hong et al.}

wooden building (Tongmyeongjeon) in Korea, because a live tree with such a long age range is rare in this country. Tongmyeongjeon is located in Seoul, and the woods used as building materials are thought to have grown at a close site to Seoul, in the center of the Korean Peninsula.

The ${ }^{14} \mathrm{C}$ concentration offset during $\mathrm{AD} 1650$ to 1850 from the IntCal data was found to be remarkably reduced compared to the corresponding previous value of $400 \mathrm{yr}$. The climate was colder during this period and the $\mathrm{CO}_{2}$ release rate of the northern Pacific Ocean occurred at a lower rate. This is in good agreement with the contention that the Little Ice Age began around AD 1650 and ended around AD 1850. The possibility that a global event can induce a regional variation of ${ }^{14} \mathrm{C}$ concentration should be noted.

\section{ACKNOWLEDGMENT}

This work was supported by the General Research Project funded by the Korea Research Council for Industrial Science \& Technology and the Ministry of Knowledge Economy of Korea.

\section{REFERENCES}

Baillie MGL. 1982. Tree-Ring Dating and Archaeology. Chicago: University of Chicago Press. 274 p.

Baillie MGL, Pilcher JR. 1973. A simple cross-dating program for tree-ring research. Tree-Ring Bulletin 33: $7-14$.

Eddy JA. 1976. The Maunder minimum. Science 192(4245):1189-202.

Hong W, Park JH, Kim KJ, Woo HJ, Kim JK, Choi HW, Kim DG. 2010a. Establishment of chemical preparation methods and development of an automated reduction system for AMS sample preparation at KIGAM. Radiocarbon 52(2-3):1277-87.

Hong W, Park JH, Sung KS, Woo HJ, Kim JK, Choi HW, Kim GD. 2010b. A new 1MV AMS facility at KIGAM. Radiocarbon 52(2-3):243-51.

Hong W, Park JH, Park WK, Sung KS, Lee KH, Park G, Kim YE, Kim JK, Choi HW, Kim GD, Woo HJ, Nam TG. 2013. Calibration curve from AD 1250 to 1650 by measurements of tree-rings grown on the Korean Peninsula. Nuclear Instruments and Methods in Physics Research B 294:435-9.

Mann ME. 2002. Little Ice Age. In: Munn T, editor. Encyclopedia of Global Environmental Change. Chichester: John Wiley \& Sons, Ltd. p 504-9.

Nakamura T, Miyahara H, Masuda K, Menjo H, Kuwana K, Kimura K, Okuno M, Minami M, Oda H, Rakowski A, Ohta T, Ikeda A, Niu E. 2007. High precision ${ }^{14} \mathrm{C}$ measurement and wiggle-match dating of tree rings at Nagoya University. Nuclear Instruments and Methods in Physics Research B 259(1):408-13.

Park WK, Lee JH. 2001. Development of tree-ring chronologies of Pinus densiflora from Mt. Sorak and dating the year of construction of the Kyunghoe-ru pavil- ion in Seoul. Journal of Korean Physical Society 39(4):790-5.

Park WK, Son BH, Han SH. 2003. Dendrochronology of wooden materials from Tongmyeongjeon of Changdeok Palace in Seoul. Journal of Architectural History 35:53-63. In Korean.

Park WK, Kim YJ, Seo JW, Lee JH, Wazny T. 2007. Tree-ring dating of Sinmu-Mun, the North Gate of Kyungbok Palace in Seoul. Tree-Ring Research 63(2): 105-9.

Reimer PJ, Baillie MGL, Bard E, Bayliss A, Beck JW, Bertrand CJH, Blackwell PG, Buck CE, Burr GS, Cutler KB, Damon PE, Edwards RL, Fairbanks RG, Friedrich M, Guilderson TP, Hogg AG, Hughen KA, Kromer B, McCormac G, Manning S, Bronk Ramsey C, Reimer RW, Remmele S, Southon JR, Stuiver M, Talamo S, Taylor FW, van der Plicht J, Weyhenmeyer CE. 2004. IntCal04 terrestrial radiocarbon age calibration, 0-26 cal kyr BP. Radiocarbon 46(3): 1029-58.

Reimer PJ, Baillie MGL, Bard E, Bayliss A, Beck JW, Blackwell PG, Bronk Ramsey C, Buck CE, Burr GS, Edwards RL, Friedrich M, Grootes PM, Guilderson TP, Hajdas I, Heaton TJ, Hogg AG, Hughen KA, Kaiser KF, Kromer B, McCormac FG, Manning SW, Reimer RW, Richards DA, Southon JR, Talamo S, Turney CSM, van der Plicht J, Weyhenmeyer CE. 2009. IntCal09 and Marine09 radiocarbon age calibration curves, 0-50,000 years cal BP. Radiocarbon 51(4): 1111-50.

Stuiver M, Polach HA. 1977. Discussion: reporting of ${ }^{14} \mathrm{C}$ data. Radiocarbon 19(3):355-63. 